\title{
Analysis of the Application of Digital Agricultural Technology in the Development of Farm Economy
}

\author{
Sijin Liu ${ }^{1}$ Zhirun Huo ${ }^{2 *}$ \\ ${ }^{1}$ School of Big Data Application and Economics, Guizhou University of Finance and Economics, Guiyang, Guizhou 550001, China \\ ${ }^{2}$ School of Economics and Management, Huzhou Normal University, Huzhou 313000, Zhejiang, China \\ DOI: $10.32629 /$ memf.v2i6.593
}

\begin{abstract}
With the significant improvement of China's science and technology level, modern information technology promotes the development of all industries and fields, which realizes the efficient use of digital resources. Digital technology is a typical example. Therefore, the construction of the agricultural system should break the traditional farm economic form and attach importance to digital construction. This provides strong support for farm economic development. This paper aims to analyze the application of digital agricultural technology in the development of the farm economy, which provides a reference for relevant research.
\end{abstract}

Keywords: digitization, agricultural technology, farm economics

\section{Introduction}

In recent years, information technology has been widely used in China's agricultural development. It relies on digital technology to accurately and visually manage agricultural information resources. This facilitates the analysis and processing of valid data and provides a decision-making basis for relevant departments. It better serves the majority of farmers to achieve the development of modern agriculture ${ }^{[1]}$. In the background of data, the farm economy is facing opportunities and challenges.

The role of information technology should be actively brought into play to realize the efficient utilization of resources and promote the farm economy to a new stage.

\section{Digital technology and modern farm economy overview}

In the current agricultural field, the essence of digital management technology application is to adopt advanced management technology. It serves agricultural construction and uses optimization and improved management methods to realize farms' sustainable, regular, healthy, and efficient operation. Combined with the development of agriculture in China, the implantation of digital management technology should involve multi-angle, and all-round management processes, such as personnel scheduling, financial data processing, basic machinery construction, and the application continues. At the same time, it is necessary to combine advanced technology with traditional agricultural mode to improve the efficiency of agricultural management and realize the normalization of comprehensive management. Finally, give full play to the complementary role of technological advantages, effectively solve the potential problems of the traditional management system, realize the optimal allocation of resources, and improve the farm's fast and efficient operation mode. In addition, digital technology should effectively improve the status of agriculture. By promoting mechanization level and technical means, it realizes the healthy and sustainable development of the agricultural industry.

As advanced technological means, Internet technology, digital technology, and storage technology promote the application of multiple industries ${ }^{[2]}$.

The age of digital technology has arrived. Automatic, intelligent generation of massive data will be fast and efficient data processing. Technical measures can implement data statistics and develop and utilize data resources to achieve the purpose of intelligent management and comprehensive efficiency. However, data information technology is not beneficial to data analysis, processing, and application as a common means. In agricultural development, agricultural digitalization technology effectively integrates and applies a variety of technologies, such as cloud computing technology and storage technology, during which algorithm theory should be optimized and improved. It thoroughly mines the practical effect of data resources. The popularization and application of agricultural information technology break the traditional agricultural form. It improves the reliability, accuracy, and security of information applications and expands the sustainable development path of the farm economy. 
Therefore, digital agricultural technology will be integrated into the development of the farm economy. Furthermore, the agricultural data application algorithm can expand the application space of farm data and boost the agricultural development system, which has important practical significance.

Nowadays, our country's agricultural economy develops regional difference characteristic is big. Therefore, the application of digital technology should be combined with the actual development of the local agricultural economy. Specifically, agricultural areas with relatively developed economic levels can strengthen the application scope of digital management technology. Furthermore, technical popularization and publicity increase the scale construction of regional farms and adopt effective technical means to promote the digitalization of agriculture. At the same time, pilot areas for digital management can be created.

This provides a practical, theoretical basis for the extension of other agricultural models. The relatively backward agricultural economy should break the situation dominated by the traditional agricultural model, coordinate a variety of advanced technological means, serve the agricultural production practice, improve the level of mechanized operation, and save human resources and material resources. During the adoption of digital technology, it is necessary to manage the internal system, attach importance to agricultural technology research and development, improve the transport capacity of agricultural products, improve the backward status of agriculture, improve the development level of the local economy and realize the overall management of agricultural industry with the support of digital management technology ${ }^{[3]}$.

\section{Digital age farm economy faces opportunities and challenges}

\subsection{Information management presents diversified characteristics}

Under the background of the data information age, the modern farm economy is highly comprehensive. Massive data makes the farm economy appear diversified. From the perspective of modern farm economic development, optimizing information management mode based on scientific management can boost the development of the new road. The application of information technology expands storage space and enriches storage media. The basic diversified features of data information face vast and complex information capacity.

The staff should have high professional skills and comprehensive quality. It should have the ability to adapt to changes and use information processing technology flexibly. It grasps the accuracy and science of data. Combining digital data and farm economy requires new ideas, new systems, and new methods under the application prospect of information construction, which provides a new kind of challenge.

\subsection{Digital management methods need to be improved}

In farm economic development, the application of digital management technology does not lie in single data processing and recording. However, in the initial stage of digital construction, the importance and necessity of digital management method should be recognized - digital management as a means to improve the efficiency of computing and analysis. Based on agricultural efficiency and precision, it provides a new way to develop the traditional farm economy using statistical data. The integration and application of information technology is the continuous optimization and improvement of algorithms.

It aims to improve statistical efficiency. This satisfies the analysis and utilization of various functions ${ }^{[4]}$. In the practice of farm economy, the data channels and approaches are broadened through advanced technology to improve the shortcomings of the traditional farm economy. It is challenging to combine the rationalization of software technology with the development of the farm economy.

\section{Analysis of problems in the application of agricultural digitization technology}

\subsection{Belittling agricultural digitization}

In the development of the modern farm economy, the introduction of information technology has achieved remarkable results. However, in practice, it is still based on the traditional farm model. The main reason lies in the lack of understanding of digital technology. The farm economy is highly integrated. It touches on everything.

Moreover, digital technology is an important branch. However, the staff will overlook this fundamental role and ignore the innovation and application of methods, lacking subjective initiative in applying digital technology by the traditional model, technological innovation, theoretical innovation, and system innovation.

Therefore, it does not ensure the combined effect of technology application. In the combination of the farm economy and digital technology, the staff is passive to accept and learn. They cannot innovate theoretical knowledge, which hinders and restricts the development of the farm economy. Therefore, it improves the practical efficiency of digital technology 
fundamentally. Based on the actual situation, it pursues a novel and scientific management path. It aims to improve the efficiency of digital farm management and maximize the advantage and value of information technology.

\subsection{Management personnel are of low professional level}

Integrating digital technology into the farm economy is a messy business. To build a high-quality, high-level, and high-quality management team, the molding and training of the talent team should improve the comprehensive management efficiency. It focuses on long-term future development to improve management ideas and methods based on the actual farm economy ${ }^{[5]}$. In practice, management fails to recognize the importance and necessity of digital technology. The construction of the talent echelon cannot deal with the relationship between talent allocation and resource management. It is unable to carry out business skills training strategically and purposefully. Especially in the actual research, the talent team can not ensure the comprehensive management effect of digital management.

\section{Digital technology improves strategy in the farm economy}

\subsection{Increase the range of information technology applications}

Modernization is significant in the development of the farm economy. At present, it is still given priority to by traditional agricultural model. This fails to bring out the actual utility of digital technology. It is necessary to pursue scientific and reasonable management strategies and maximize technical and management efficiency based on maintaining the regular operation of the farm economy. The farm economy faces both opportunities and challenges. It should be combined with specific problem processing and use of data. In data collection and collection, information collection technology should be used reasonably to break the limitation. It is worth noting that modern information technology provides unlimited possibilities for farm economic development. It actively responds to the technological background and uses information technology. In addition to analyzing various sample data, we should also pay attention to the analysis and calculation process. Therefore, to develop the farm economy better, we should enhance the application scope of information technology and provide a broader space for development.

\subsection{Improve the accuracy of information management}

Information processing is not paid attention to in the traditional agricultural model, and information collection is characterized by low speed. The integration of information technology into agricultural development can enrich primary data resources and innovate the farm economy's development model. Relying on a network means data processing and application in the agricultural field can be realized, especially in creating a statistical database, which can save manpower and material resources, and cost ${ }^{[6]}$. The farm economy covers a large amount of essential data processed and analyzed by information technology. Through in-depth mining of data information, more accurate statistical results can be obtained. Of course, the integration of information technology into the farm economy makes data more standardized and unified. In addition to meeting the basic mass storage needs, it implements the process of data accumulation. At the same time, the use of computing and information systems as auxiliary means to realize the sharing of resources, give full play to the function of resource optimization, boost the new trend of farm economic development.

\subsection{Focus on building a modern digital technology system}

The modern farm economy breaks the pattern of traditional agriculture and has the essential characteristics of complexity and comprehensiveness. When using digital technology, it is no longer a traditional and separate work.

It is closely related to the farm economy.

It is combined with the work content of the enterprise. Information technology, as the primary means and management methods, maximize the actual utility of modern technology. At the same time, the practicability of digital technology in the farm economy should be continuously enhanced. In the process of resource data, in addition to doing essential work, we should innovate ideas, change the traditional agricultural model, eliminate the barriers in various work links, and summarize and analyze the problems and shortcomings in the application of technology in time. Finally, the staff should create corresponding solutions from a global construction perspective. With the farm economy as the focus, it attaches importance to the practical application of digital technology.

\subsection{Training modern advanced personnel}

The development of the modern farm economy can not leave the binding force of talent. Digital technology theory is integrated into farm operation form, and technology is used to urge work rectification [7-8]. For staff, business skills training should be strengthened and expand the talent team and into the technical learning. It makes the efficiency of the work come 
out. In the process of farm economic development, breakthroughs have been made under the impetus of modern technology. However, in reality, many brain drain restricts and hinders agricultural development, especially the outdated knowledge and methods mastered by staff cannot meet the needs of long-term development, so attention should be paid to the improvement of personnel management ability. Therefore, shaping and cultivating a modern talent team should be a fundamental goal, implementing systematic business training for digital technical personnel and enhancing practical application ability. This gives them a combination of skills and qualities to serve the modern farm economy better.

\subsection{Create agricultural farm statistics information application system}

\subsubsection{Data acquisition of farm economy statistics digitization system}

Digital technology is used to serve the on-site farm economy. Creating a farm management system is the foundation and prerequisite. Attention should be paid not only to promoting professional machinery construction but also to strengthening professional agricultural guidance. Relevant departments need to strengthen supervision and improve rules and regulations to ensure the legality and effectiveness of technology application. It should be supported by information technology, with the accuracy and timeliness of information as the basis to meet the fast and efficient transmission of information, realize the optimal allocation of resources, and avoid economic and resource waste.

In establishing the farm economic statistics digital system, the following three points should be paid attention to in the data collection and collection. First, for information storage, we should use technical means to summarize and sort out the farm information. In addition to creating network data scale, ensure data integrity and precision. It takes farm data (yield, income, etc.) as indicators. Create monthly, quarterly, and annual statistical reports. While improving the all-around performance, it also saves much workforce and material resources and gives full play to the auxiliary role of technology. Second, the analysis and processing of big farm data require an accurate grasp of digital technology—comprehensive handling of various work, such as output statistics, agricultural transportation, etc. In the traditional farm economic model, there is incomplete data information over the years.

Especially some basic information, with the characteristics of incomplete records. In this regard, digital technology should be used to deal with some interactive and complex agricultural information, realize the visualization and exemplary management of the farm economy, and achieve the expected technical effects and goals ${ }^{[9]}$. Thirdly, when dealing with agricultural information collection and collection and strengthening daily communication of staff, information technology should also be adopted. Create a professional information exchange and storage platform for the feasibility of data management to create suitable conditions. Departments concerned should attach great importance to the process of agricultural development. The actual situation creates a database or website supported by technology to meet the efficient use and management of farm data information.

\subsubsection{Index expression and realization of farm economy statistics digital system}

Use digital technology to develop the modern farm economy. In the process of agricultural production, the digital system of farm economic statistics is built. Meanwhile, Access database technology is adopted to strengthen Hisense information data storage function. In order to develop a network application system, ASP dynamic website should be used as a reference to playing the role of data and analysis, such as scatter chart analysis function, economic growth rate function. This technology is intuitive and can objectively reflect the development of agricultural economy data, such as agricultural economic input, economic growth rate, etc., to meet the multi-terminal sharing function under the network. This creates favorable conditions for the platform of an integrated, integrated agricultural economy network.

The variance contribution rate is the main index of digital farm construction. This concept refers to the process of change of original indicator information. Generally speaking, if the cumulative variance contribution rate is between $85 \%$ and $105 \%$, the principal component can represent the relationship of variables in the analysis of the original indicators. In the actual analysis process, the correlation coefficients of load factors in each principal component should be sorted to get the yield capacity of farm output. For example, to better improve the economic level of the modern farm, 597 farms took the lead in the application of digital technology in the Heilongjiang Reclamation area. It creates digital agricultural information systems. As the most representative demonstration base, it combines economic benefits with environmental protection and uses digital technology. Starting from the actual agricultural development, the data of good idea farm should be counted, statements should be created to form the institutional basis, agricultural information resource database should be created, agricultural development should be actively promoted, and reasonable development and application of data resources should be realized ${ }^{[10]}$.

\section{Conclusion}

By integrating digital technology into the development of the farm economy, this paper adopts advanced information 
technology means to enhance the application scope of information technology, build a modern digital technology system, and create an agricultural farm statistics information application system. Therefore, digital technology and the modernization of the farm economy are extraordinarily significant to improving the agricultural level.

\section{References}

[1] Yu Xiaolin. Study on the application of digital agricultural technology in the development of farm economy [J]. Agricultural technology service, 2017, 34(1): 182.

[2] Peng Bo, Zhang Meng. Research on application of digital Design technology in agricultural machinery design [J]. Southern Agriculture, 2020, (29): 174-175.

[3] Ren Chenghao, Zheng Xiqing, Duan Xueqin. Preliminary study on agricultural informatization application system based on "smart radio and television" — A Case Study of "Smart Farm" [J]. Agriculture Today, 2019, 000(006): 19-20.

[4] Chen Jing, Zeng Ya. Empirical analysis of integrated development of leisure agriculture and rural tourism — A Case study of Leisure farm development in Dalian city, Liaoning Province [J]. Journal of Shenyang Agricultural University (Social Science Edition), 2019, (03): 16-20.

[5] Liang Zhu. Digital Design technology and its application analysis in agricultural machinery design [J]. Internal Combustion Engines and Accessories, 2019, 282(06): 227-229.

[6] Zhao Jianshe, Yang Qiaoyun, Xie Kaiquan, et al. Analysis of Ecological Benefit of "Rice-duck Farming" Model and Its Economic Benefit in Ecological Farm [J]. Journal of Agricultural Science and Technology, 2019, (11): 149-156.

[7] Wu Chunhua, Pan Xinwei. Brave to undertake the task of digital transformation and Development: A record of the development of digital agriculture in Changshuihe Farm in Heilongjiang Province [J]. Management of Farm Economy, 2019, 283(10): 67-69.

[8] Wang Benfeng. Application status of agricultural mechanization and its role in agricultural production [J]. Hubei Agricultural Mechanization, 2019, 000(009): 3.

[9] Ni Hao, Liu Zhimin. Research on adoption behavior and influencing factors of Internet agricultural technology in family farms: A case study of 270 family farms in nine cities of Jiangsu Province [J]. Nanjing Social Science, 2019, 000(002): 34-40.

[10] Chen Chun Feasibility analysis of sharing farm model in Putian Mountain area -- A case study of Dayang Township [J]. Rural families, 2020, 241(01): 32-33. 